\title{
PHOTOCHEMISTRY AND STRUCTURE IN NUCLEIC ACIDS
}

\author{
A. A. LAMOLA \\ Bell Laboratories. Murray Hill. New Jersey 07974, USA
}

\begin{abstract}
Research into the photochemistry of biopolymers has increased in scope and scale to such an extent in recent years that it has become necessary to limit this review to a consideration of photochemical approaches to the structure of polynucleotides. Treatment proceeds from the constituent monomers to the polymer to emphasize the changes in photophysical and photochemical properties of the monomers when incorporated into the polymer. Studies of the mechanisms and structural requirements for the photoreactions in polynucleotides are complicated by low yields of reactions and the essential lack of luminescence from nucleotides in aqueous solution near room temperature. Contrariwise, the use of photochemical yields in an empirical way to indicate occurrence of conformational changes has been successful. The most successful use of photochemical reactions in polynucleotides has been for making specific structural modifications for structure-function studies.
\end{abstract}

\section{INTRODUCTION}

Interest and activity in the photochemistry of biopolymers have continued to increase and the topic has for some years been too large to review in one lecture. I have chosen to review that part of the activity concerned with photochemical approaches to the structure of polynucleotides because it is a reasonable portion to review here and because it fits in well with a large fraction of the photochemical studies on synthetic polymers.

Photochemical processes in biopolymers and in synthetic polymers have been investigated for the same reasons. These include: (1) the challenge to understand the photochemistry of a collective system; (2) the opportunity to obtain structural information; (3) the opportunity to modify the polymer in a specific way; (4) the elucidation of the basis for photoinactivation of function (photodegradation in the case of synthetic polymers); and (5) the elucidation of mechanisms of photobiological function (imaging processes and the like in the case of synthetic polymers). Early interest in the photochemistry of nucleic acids stemmed from the findings that the DNA (RNA for RNA viruses) of microorganisms is the most sensitive target for their u.v.-photoinactivation and for u.v.-induced mutagenesis. More recently, photochemical studies of polynucleotides (especially various RNAs) aimed at elucidating secondary and tertiary structure (conformation) and for making modifications useful in structure-function studies have become more 
numerous. The results to date have revealed that the relationship between structure and photochemical reactivity in polynucleotides is complex. On the other hand, useful techniques for specific photochemical modification of nucleic acids are being developed rapidly.

My treatment will proceed from the constituent monomers to the polymer in order to emphasize the changes in the photophysical and photochemical properties of the monomers when they are incorporated into the polymer. Of course, experimentation has not proceeded in this fashion. Work at the level of monomers and at the polymer level has proceeded simultaneously, usually in different laboratories and with different points of view. For a complete view of the field of nucleic acid photochemistry one should read several of the recent reviews which are listed as references 1 to 7 .

\section{Structures, nomenclature and abbreviations}

The chemical structures of the common bases and three of the 'odd' bases which occur naturally in nucleic acids are given below.<smiles>[R]n1cnc2c(N)ncnc21</smiles>

A<smiles>Cn1ccc(=O)[nH]c1=O</smiles>

U<smiles>Cn1ccc(=S)[nH]c1=O</smiles><smiles>Cn1cnc2c(=O)[nH]c(N)nc21</smiles>

G<smiles>Cn1ccc(N)nc1=O</smiles>

C<smiles>Cc1c[nH]c(=O)[nH]c1=O</smiles>

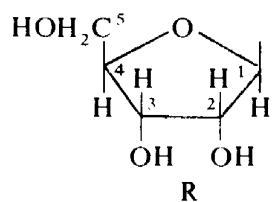<smiles>[R17]n1cc(C)c(=O)[nH]c1=O</smiles>

$\mathrm{T}$<smiles>COC(=O)NC(CI)C(=O)OC</smiles><smiles>CCc1c(C)nc2n(C)c3c(ncn3C)c(=O)n12</smiles>

$\mathrm{Y}$

The following abbreviations are used for the ribonucleosides: $\mathrm{A}$, adenosine; $\mathrm{G}$, guanosine: $\mathrm{T}$, thymidine (deoxyribonucleoside); $\mathrm{C}$, cytidine; $\mathrm{U}$, uridine ; $\psi$, pseudouridine; and, $\mathrm{s}^{4} \mathrm{U}$, 4-thiouridine. The corresponding deoxyribonucleosides are indicated by the prefix $\mathrm{d}$ as in $\mathrm{dA}$, deoxyadenosine, 
except for T, which already refers to the deoxyribonucleoside. Unless otherwise indicated, the mononucleotide indicated by the suffix MP, as in AMP, adenosine monophosphate, means that the phosphate ester linkage is at the 5-position of the sugar group. Dinucleotides represented by the formula $\mathrm{XpY}$, as for example, ApC, are $3^{\prime} \rightarrow 5^{\prime}$; that is, the first nucleoside is connected at the $3^{\prime}$-position and the second at the $5^{\prime}$-position.

Homopolymers are abbreviated poly $\mathrm{rX}$ or poly $\mathrm{dX}$ for the polyribonucleotide and polydeoxyribonucleotide, respectively, as, for example, poly dA is polyadenylic acid. Double-stranded polynucleotides are indicated by the use of a colon, as, for example, poly $\mathrm{rG}: \mathrm{rC}$, in which one strand contains only guanine and the other strand cytosine. Poly(dAT):dAT represents the double stranded polymer in which each strand contains adenine and thymine in an alterating sequence.

In Watson Crick base pairing, such as found in DNA, G is hydrogenbonded to $C$, and $A$ with $T$, or $A$ with $U$ in the case of RNA.

\section{PHOTOPHYSICS OF NUCLEIC ACIDS ${ }^{8}$}

\section{Monomers}

Some excited state parameters for the ordinary mononucleotides are are given in Table 1 . The striking feature common to all of them is that electronic relaxation from the lowest excited singlet state is predominantly radiationless and occurs directly to the ground state despite the large electronic energy gaps $(\sim 4 \mathrm{eV})$. (Formation of unstable photoisomers is not a viable explanation.) While radiationless decay is fast at $80^{\circ} \mathrm{K}$, it becomes even faster as the temperature rises so that the excited singlet state lifetimes are extremely short $(\sim 10 \mathrm{ps})$ at room temperature in water solution. The rates of intersystem crossing in the pyrimidines also increase with temperature such that, in some cases, the triplet yields are higher at room temperature than at $80^{\circ} \mathrm{K}$. For TMP and UMP at room temperature both the fluorescence yields ${ }^{9}$ and the triplet yields increase with decreasing wavelength of the exciting light through the first absorption band. This can be explained if fluorescence and intersystem crossing from upper vibrational levels of the excited singlet state contribute significantly to the total fluorescence and intersystem crossing yields. Some factors affecting photophysical processes such as $\mathrm{pH}$ have been explained. Some remain unexplained. For example, thymine does not undergo intersystem crossing in dilute solution at $80^{\circ} \mathrm{K}$ but sizeable triplet yields are obtained in concentrated solutions in which aggregation takes place.

Molecular orbital descriptions of the low-lying excited states of some of the nucleotides have been developed. The best described is thymine. Discussion of these descriptions is beyond the scope of this presentation. Suffice it to say that all of the excited states observed in luminescence $\left(80^{\circ} \mathrm{K}\right)$ are $\pi, \pi^{\star}$ states except for the fluorescent state of AMP which may be $n, \pi^{\star}$. Low-lying $n, \pi^{\star}$ states are expected for all the nucleotides and, although they are not observed directly, they are no doubt important in controlling relaxation paths and rates. 
Table 1. Excited state paramete

\begin{tabular}{lcccc}
\hline Material & $\begin{array}{c}{ }^{1} \mathrm{E} \\
\left(10^{3} \mathrm{~cm}^{-1}\right)\end{array}$ & $\begin{array}{c}\varphi_{\mathrm{F}} \\
\left(80^{\circ} \mathbf{K}\right)\end{array}$ & $\begin{array}{c}{ }^{1} \tau\left(80^{\circ} \mathbf{K}\right) \\
(\mathbf{n s})\end{array}$ & $\begin{array}{c}\varphi_{\text {isc }} \\
\left(80^{\circ} \mathbf{K}\right)\end{array}$ \\
\hline A (pH 7) & 35.2 & 0.01 & 2.8 & 0.02 \\
A (pH 2) & 34.8 & $\sim 0$ & - & $\sim 0$ \\
G(pH 7) & 34.0 & 0.13 & -5 & 0.15 \\
C (pH 7) & 33.7 & 0.05 & - & 0.03 \\
T (pH 7) & 34.1 & 0.16 & 3.2 & -0 \\
T (pH 12) & 34.35 & 0.24 & 2.9 & 0.15 \\
U (pH 7) & 34.9 & -0.01 & - & - \\
U (pH 12) & 35.0 & 0.005 & - & - \\
\hline
\end{tabular}

Data for the $5^{\prime}$-nucleoside monophosphates as dilute solutions in EGW $\left(80^{\prime \prime} \mathrm{K}\right.$ ) or $\mathrm{D}_{2} \mathrm{O}$ (room temperature). The exciting resonance spectra. The room temperature data were obrained using $\mathrm{Eu}^{3+}$ ions as excited state probes. The $\mathrm{pD}$ in the latter unpublished reports.

\section{Dinucleotides and polynucleotides}

Interactions between the bases in a polynucleotide modify the excited states and associated relaxation processes and can give rise to electronic energy transfer. These interactions are short range, the most slowly varying ones going as the inverse cube of the distance between the interacting centres. Thus, pair-wise interactions between neighbouring bases are the most important, and dinucleotides become useful models for single-stranded polynucleotides. This is especially so because the bases in many dinucleotides tend to 'stack' so that their relative positions are similar to those found, for example, in DNA.

Except for hypochromicity the absorption spectra of dinucleotides and polynucleotides differ little from those of mixtures of the constituent monomers. In contrast, the fluorescent emissions $\left(80^{\circ} \mathrm{K}\right)$ from many dinucleotides and polynucleotides are greatly red-shifted $\left(\sim 5000 \mathrm{~cm}^{-1}\right)$ from that of a mixture of constituent monomers. Some examples are shown in Figure 1. The shifts are at least an order of magnitude greater than those found in absorption. A necessary but not sufficient condition for the red shift in the fluorescence is that the bases are stacked. These observations are best explained by the formation of an excited state complex (exciplex) between neighbouring stacked bases.

Some dinucleotides give both monomer and exciplex-like fluorescence spectra. This has been attributed to conformational heterogeneity. The way in which the two bases are linked together has a large effect on the exciplex fluorescence. Thus, ApC and $\mathrm{CpA}$ give different spectra which are probably not indicative of single geometries (Figure l). The sensitivity of exciplex formation towards structure is further demonstrated by the fact that poly $\mathrm{dA}$ and $\mathrm{A}-\left(\mathrm{CH}_{2}\right)_{3}-\mathrm{A}$ (two adenines linked at their 9-positions by a trimethylene bridge) give excimer spectra, ApA and poly rA give monomer-like fluorescence. Unfortunately there is not sufficient knowledge of the electronic structures of the excited states of the nucleotides to allow conclusions about geometry from the characteristics (wavelength, yield, etc.) of exciplex fluorescence in dinucleotides, except that some degree of stacking is necessary for exciplex formation.

The decay of the singlet exciplexes at $80^{\circ} \mathrm{K}$ is predominantly non-radiative 
PHOTOCHEMISTRY AND STRUCTURE IN NUCLEIC ACIDS

f the common mononucleotides

\begin{tabular}{cccccc}
$\begin{array}{c}\left(1-\varphi_{\mathrm{F}}-\varphi_{\text {isc }}\right) \\
\left(80^{\circ} \mathrm{K}\right)\end{array}$ & $\begin{array}{c}{ }^{3} \mathrm{E} \\
\left(10^{3} \mathrm{~cm}^{-1}\right)\end{array}$ & $\begin{array}{c}\varphi_{\mathrm{P}} \\
\left(80^{\circ} \mathrm{K}\right)\end{array}$ & $\begin{array}{c}{ }^{3} \tau\left(80^{\circ} \mathrm{K}\right) \\
(\mathrm{s})\end{array}$ & $\begin{array}{c}\varphi_{\text {isc }} \\
\left(300^{\circ} \mathrm{K}\right)\end{array}$ & $\begin{array}{c}{ }^{1} \tau\left(300^{\circ} \mathrm{K}\right) \\
(\mathrm{s})\end{array}$ \\
\hline 0.97 & 26.7 & 0.015 & 2.4 & $4 \times 10^{-4}$ & $1 \times 10^{-12}$ \\
$\sim 1.0$ & 27.35 & $\sim 0$ & 1.75 & $\overline{-}$ & - \\
0.72 & 27.2 & 0.07 & 1.3 & $5 \times 10^{-4}$ & $4 \times 10^{-12}$ \\
0.92 & 27.9 & 0.01 & 0.34 & $1.5 \times 10^{-3}$ & $4 \times 10^{-12}$ \\
0.84 & 27.3 & $\sim 0$ & 0.35 & $8 \times 10^{-3}$ & $2 \times 10^{-11}$ \\
0.61 & 27.0 & 0.03 & 0.45 & & - \\
-0.99 & 27.4 & -0 & 0.55 & $7 \times 10^{-3}$ & $2 \times 10^{-11}$ \\
-0.99 & 28.4 & 0.002 & - & - & - \\
\hline
\end{tabular}

wavelength was $265 \mathrm{~nm}$ throughout. The low temperature ditlit were extracted from lumunescence spectra and elettron spin experiments was about five. These data were obtained at the Bell lithoratories and are collected from several pubished and

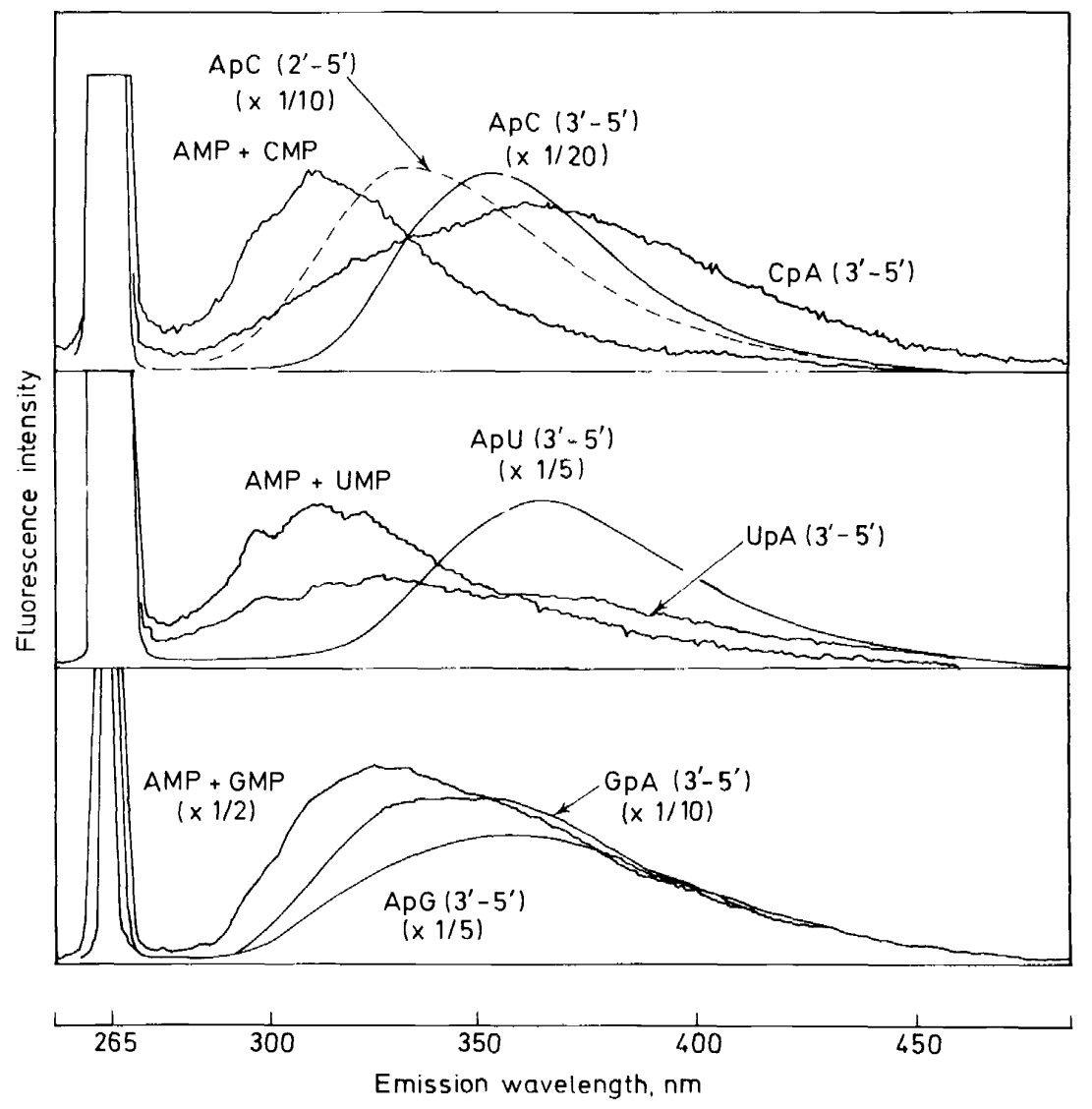

Figure 1. Fluorescence spectra excited at $265 \mathrm{~nm}$ from various dinucleotides in buffered ethylene glycol-water glasses at $80^{\circ} \mathrm{K}$ compared to the spectra obtained from equimolar mixtures of the mononucleotides. 


\section{A. A. LAMOLA}

as is observed for the isolated monomers. While exciplex formation can compete favourably with decay of monomer singlet states at $80^{\circ} \mathrm{K}$ (in many cases only exciplex emission is observed), the much faster decay of the monomer states at room temperature makes this much less likely at the higher temperature.

Intersystem crossing $\left(80^{\circ} \mathrm{K}\right)$ occurs from the exciplex in those dinucleotides which form an exciplex. and is about as efficient as in the monomers. The phosphorescence $\left(80^{\circ} \mathrm{K}\right)$ from dinucleotides is almost always virtually identical to that of the component with the lowest lying triplet-triplet state. Thus only very weak interactions between the bases occur in the triplet state.

In addition to base stacking, hydrogen bonding between complementary bases in double stranded polynucleotides has an important effect upon the electronic relaxation. Thus, fast relaxation processes occur in $\mathrm{G}-\mathrm{C}$ and $\mathrm{A}-\mathrm{U}$ base pairs which quench the excited singlet state; e.g the fluorescence yield $\left(80^{\circ} \mathrm{K}\right)$ from the copolymer poly $(\mathrm{dG}): \mathrm{dC}$ is an order of magnitude smaller than that of a mixture of the two single stranded homopolymers. Proton movement, perhaps even the production of the tautomeric base pairs by double proton transfer, may be involved in the quenching mechanism. No such quenching occurs in $\mathrm{A}-\mathrm{T}$ base pairs.

A T-T exciplex has not been observed and the exciplexes in poly dA and poly (dAT):dAT show fluorescence maxima at 350 and $355 \mathrm{~nm}$, respectively. Since hydrogen-bonded $\mathrm{G}$ and $\mathrm{C}$ are expected to contribute little, the fluorescence spectrum of DNA, which is very similar to that of poly (dAT) : dAT (Figure 2) may be rationalized as originating from neighbouring $\mathrm{A}$ and $\mathrm{T}$ bases on the same strand.

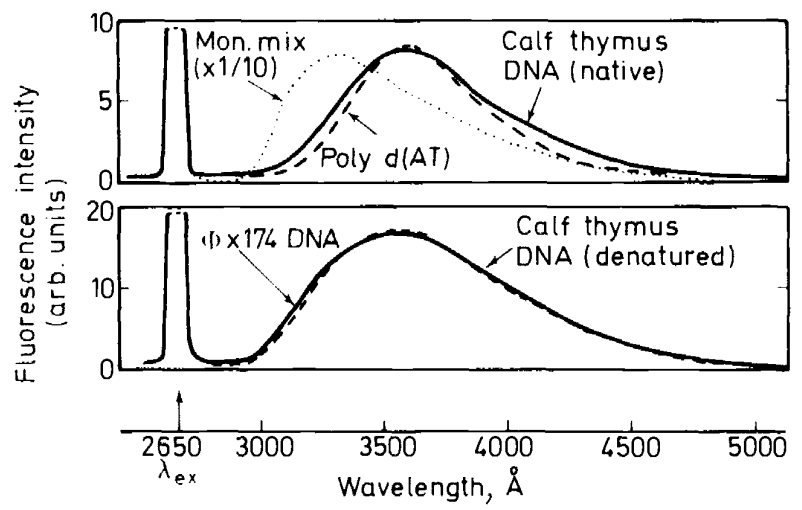

Figure 2. Top: Fluorescence spectra excited at $265 \mathrm{~nm}$ of poly(dAT):dAT. and calf thymus DNA at $80 \mathrm{~K}$ compared to the spectrum from an equimolar mixture of T, C, G and A. Bottom: Fluorescence spectra of two single stranded DNAs.

Heat denatured DNA or single-stranded DNA from phage $\Phi \times 174$ (Figure 2) show exciplex and monomer fluorescence indicating that base stacking is maintained for the most part.

Because of triplet energy migration (see below) the phosphorescence spectra of polynucleotides are expected to be characteristic of the constituent 


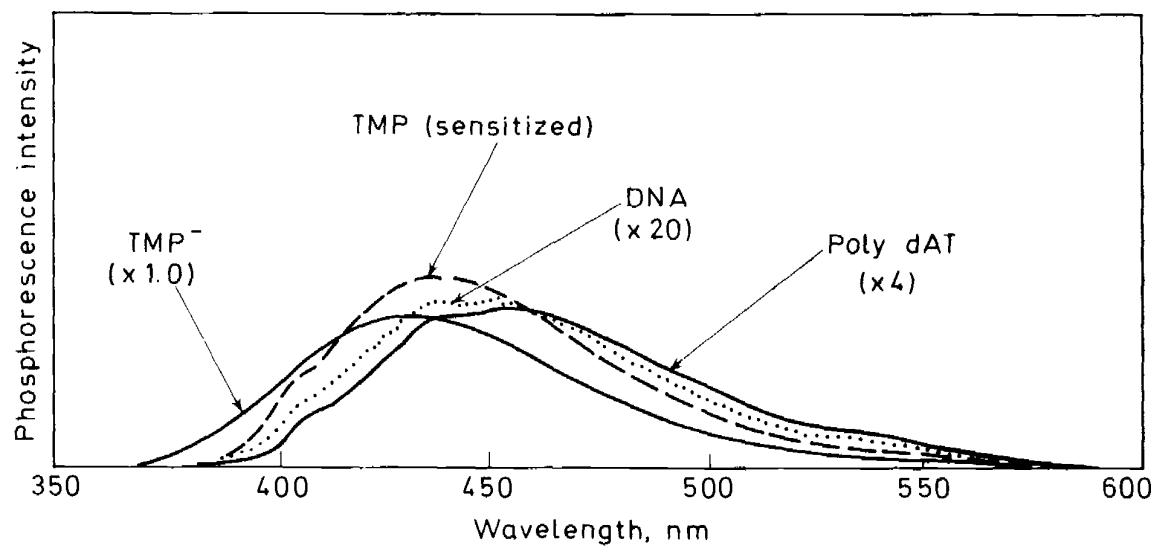

Figure 3. Phosphorescence spectra from frozen solutions $\left(80^{\circ} \mathrm{K}\right)$ of DNA, poly(dAT):dAT, TMP at $\mathrm{pH} 12\left(\mathrm{TMP}^{-}\right)$and TMP at $\mathrm{pH}$ 7. The latter was obtained using acetone as a triplet sensitizer.

base with the lowest triplet level. The phosphorescence from poly (dAT):dAT (Figure 3) is thymine-like; whereas that from poly AU is adenine-like. The weak phosphorescence from DNA originates from the thymine triplet state (Figure 3).

\section{Excitation transfer in polynucleotides}

Calculations of the rates of Förster (vibrational relaxation) transfer of singlet excitation between bases in the DNA geometry using the spectroscopic data for the mononucleotides predict that only one or two transfers to a neighbouring base can occur during the donor lifetime at $80^{\circ} \mathrm{K}$. At room temperature where the lifetimes are much shorter the transfer probability should be even lower. The low rates $\left(\sim 10^{9} \mathrm{sec}^{-1}\right.$ at $\left.80^{\circ} \mathrm{K}\right)$ can be blamed on poor spectral overlap since the electronic interactions are substantial $\left(\sim 500 \mathrm{~cm}^{-1}\right)$. Thus, before-vibrational-relaxation rates are estimated to be much greater, $10^{11}$ to $10^{14} \mathrm{sec}^{-1}$ (excitation wavelength $265 \mathrm{~nm}$ ), and could, in the fastest cases, compete with vibrational relaxation.

Consistent with calculations, singlet excitation migration in several polynucleotides (e.g. poly $\mathrm{rA}$ ) which have been examined appears to be of very short range (two or three bases).

The qualitative dependence of the fluorescence intensity $\left(80^{\circ} \mathrm{K}\right)$ from DNAs of various AT contents indicates that quenching in GC base pairs occurs at the singlet level at a rate faster than the (after-vibrational-relaxation) singlet transfer rates. This notion coupled with the idea that exciplex formation is also fast compared to energy transfer has been used to interpret the dependence of the DNA triplet yield $\left(80^{\circ} \mathrm{K}\right)$ on AT content as follows. Light of $265 \mathrm{~nm}$ wavelength is absorbed with equal probability by AT and GC base pairs. Before-vibrational-relaxation transfer allows excitation to migrate and become localized (by vibrational relaxation) at sites proportional to the trapping efficiencies which are in the ratio one to four for AT and GC pairs, respectively. The excitation in GC sites is mainly quenched. 


\section{A. A. LAMOLA}

Excited AT pairs can give exciplexes (with neighbours) and eventually the thymine triplet. Quenching in GC pairs and exciplex formation may contribute to the initial localization processes.

Triplet excitation migration $\left(80^{\circ} \mathrm{K}\right)$ in DNAs of comparable AT and GC contents appears to be of short range ( $\sim 5$ bases). The range is not lifetime limited since the intensity of phosphorescence is reduced when quenchers are added but the lifetime remains constant. Estimates of triplet transfer rates between neighbouring bases in within base pairs have yielded a model which qualitatively explains the observations ${ }^{10.11}$. The essential feature of the model is that the transfers $\mathrm{T} \rightarrow \mathrm{G}, \mathrm{T} \rightarrow \mathrm{C}$ and $\mathrm{T} \rightarrow \mathrm{A}$ along the same strand as well as interstrand transfers should rarely occur while for the other 13 possible nearest neighbour combinations the hopping time is fast compared to the decay time. All the rates increase at room temperature so that despite the shorter triplet lifetime the range of triplet migration is expected to be a little larger ( $\sim 1020$ bases). Of course, the triplet excitation resides mostly on the thymines.

Long range $(\sim 100)$ bases for triplet migration are found for poly $\mathrm{rA}$ and poly (dAT):dAT. In both cases the range is limited by unknown trapping or blocking sites which may be conformational distortions.

While it appears that excitation transfer in polynucleotides is dependent upon composition, sequence and conformation, the processes are sufficiently complex that it is unlikely that studies of excitation migration among the common bases would be useful in structure determination.

\section{Odd bases}

tRNAs contain several 'odd' nucleotides in addition to the common nucleotides. The structures of three of these are given above. An important feature of many of the odd nucleotides is that they absorb to the red of the common nucleotides which permits one to excite them exclusively. Furthermore, a few of the odd nucleotides fluoresce with good intensity in water at room temperature. Thus, since the positions of these odd nucleotides in the primary sequence of the tRNAs are frequently known, they become very useful for spectroscopic and photochemical studies of tRNA structure and function.

One odd base which has been particularly useful in this way is the so-called $Y$ base of phenylalanyl tRNA of baker's yeast. The Y base is found immediately adjacent to the 3 -end of the anticodon (Figure 4). The $\mathrm{Y}$ base has an absorption peak at $315 \mathrm{~nm}$, well to the red of the other bases in phenylalanyl tRNA, and exhibits a fluorescence peaked near $440 \mathrm{~nm}$ with a yield of about 0.1 . Eisinger and co-workers have used this fluorescence to demonstrate a $\mathrm{Mg}^{2+}$-dependent conformational change in the vicinity of the $\mathrm{Y}$ base ${ }^{12}$ and to obtain equilibrium constants for the binding of various codons to the tRNA ${ }^{13}$. In both cases shifts and yield changes of the $\mathrm{Y}$ base fluorescence were the experimental observables. Beardsley and Cantor measured the efficiency of singlet energy transfer from the $Y$ base to dye molecules covalently attached to the CCA end of the tRNA molecule ${ }^{14}$. Using the unusual assumptions connected with employing Förster transfer theory and some necessary corrections in the data, they were able to estimate the distance between the $\mathrm{Y}$ base and the dye moieties to be 40 to $60 \AA$. While the error 


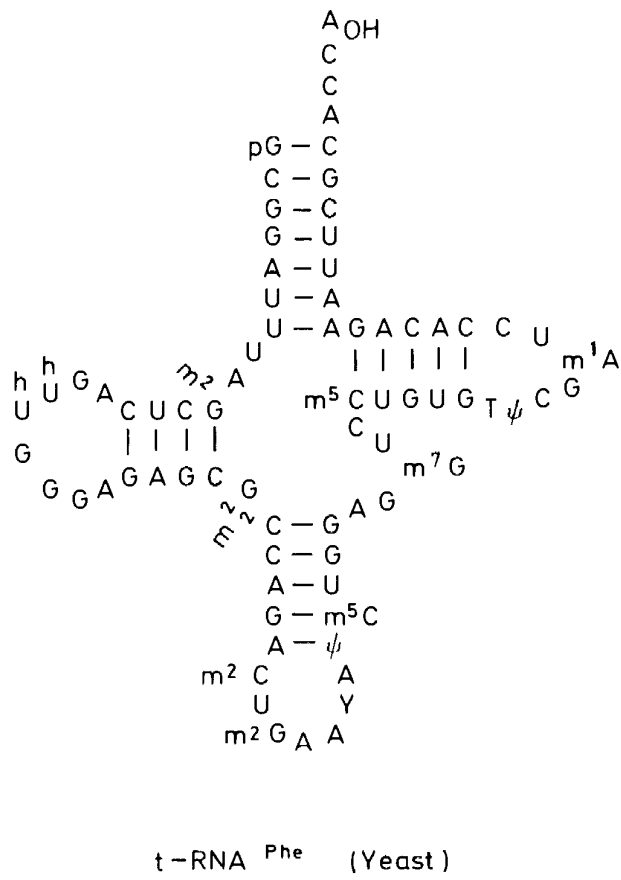

Figure 4. The cloverleaf structure for phenylalanine tRNA from baker's yeast. The $\mathrm{Y}$ base is adjacent to the anticodon (GAA) (bottom loop). The numbering starts at the $\mathrm{G}$ end and goes to the CCA end. In several tRNAs there is a $\mathrm{s}^{4} \mathrm{U}$ at position 8 which becomes bonded to a $\mathrm{C}$ at position 13 upon irradiation.

limits are quite large this result excluded several models for the three dimensional structure of the tRNA. These experiments remain the most direct experimental measure of an intramolecular distance in a tRNA.

\section{PHOTOCHEMISTRY OF NUCLEIC ACIDS}

Whereas ionizing radiation and the attack of singlet oxygen and many chemical reagents lead to reactions of both the purine and pyrimidine bases, the action of u.v. light $(240-320 \mathrm{~nm})$ leads chiefly to chemical modification of the pyrimidines. Except under special circumstances photochemical yields are low $(\sim 0.01)$ - probably a necessary property of the components of the carrier of genetic information.

Unimolecular photorearrangement reactions are unimportant except for some of the rare nucleotides like pseudouridine which will be mentioned later. The two most important reactions of the common pyrimidines under normal circumstances are photohydration and photodimerization to give cyclobutane derivatives.

Both of these reactions involve the 5-6 carbon-carbon double bond in the pyrimidines. In the photohydration reaction water is added across the double 


\section{A. A. LAMOLA}

bond; this reaction occurs for uracil and cytosine and their derivatives but does not occur for thymine derivatives. The cyclophotodimerization involves the addition of the double bonds of two pyrimidines to give cyclobutanes; all the possible homodimers and cross dimers involving uracil, thymine and cytosine can be produced in this way.<smiles>O=c1cc[nH]c(=O)[nH]1</smiles><smiles>O=C1CC(O)NC(=O)N1</smiles><smiles>Nc1cc[nH]c(=O)n1</smiles><smiles>[C-]C#C</smiles><smiles>NC1=NC(=O)NC(O)C1</smiles><smiles>Cc1c[nH]c(=O)[nH]c1=O</smiles><smiles>CC12C(=O)NC(=O)NC1C1NC(=O)NC(=O)C12C</smiles>

Photocondensation of two pyridines can also occur by way of photocycloaddition involving the 5,6-double bond of one and a $\mathrm{C}=\mathrm{O}, \mathrm{C}=\mathrm{N}$ or $\mathrm{C}=\mathrm{S}$ group on the other followed by hydrolytic ring-opening of the fourmembered ring.

All three classes of pyrimidine photoreactions occur in polynucleotides and are sensitive to the polymer structure.

\section{Photodimerization of pyrimidines}

There are many examples of photocycloaddition of two carbon-carbon double bonds in the photochemical literature. For example, many simple olefins and cyclic enones form dimers and heterodimers in this manner. In all of these cases including the photodimerization of thymine there is no evidence for complicated multistep mechanisms involving free-radical or charged intermediates. On the contrary, the formation of the two new carbon-carbon bonds often appears to be concerted. Sequential formation of the two new bonds occurs in other cases especially when the triplet state is the excited state precursor.

With respect to the photodimerization of the common pyrimidines the following questions may be raised: Which excited state precursor(s) are involved? What governs which isomers are formed? What factors govern quantum efficiency?

It is convenient to discuss these questions with respect to five situations: (1) dilute solutions of thymine and uracil; (2) concentrated solutions; (3) aligned monomer pairs; (4) dinucleotides: and (5) polynucleotides. 
The intermolecular photodimerization of thymine and uracil and derivatives in sufficiently dilute solutions $\left(<10^{-2} \mathrm{M}\right.$, depending on the solvent) where no ground state association occurs, demands the triplet state $\left({ }^{3} \tau \approx 10^{-6}\right.$ sec) as an intermediate because the excited singlet state does not live long enough $\left({ }^{1} \tau \leqslant 10^{-11} \mathrm{sec}\right)$ to take part in a bimolecular reaction. Triplet quenching experiments have shown that this is indeed so ${ }^{15,16}$.

In general all four configurational isomers are formed in solution. The ratio of isomers formed depends on the particular derivative and upon the solvent used. No definitive explanation of the observed product ratios exists. One complicating factor is that not all reactive encounters between a triplet thymine molecule and a ground state molecule lead to a dimer. On the contrary Wagner and Bucheck ${ }^{17}$ have shown that self-quenching is the main result of such interactions for thymine in acetonitrile.

As the concentration of the thymine derivative is increased a dramatic increase in the specific rate of dimer formation occurs when ground state association (stacking) begins. A good deal of experimental data has been obtained for 1,3-dimethylthymine (DMT). Lisewski and Wierzchowski ${ }^{18}$ have shown that in the high concentration range triplet quenchers are ineffective at quenching the dimerization of DMT in water. They measured the concentration and temperature dependence of the quantum yield for dimer formation and analysed the results in terms of the ground state association model and found a value for the association constant in excellent agreement with values obtained by osmometry for pyrimidine bases. Extrapolation of their yield data to infinite DMT concentration gives $\varphi_{\hat{\mathrm{TT}}}[\mathrm{DMT}]_{\infty}=0.125$ in good agreement with the dimer yield in DMT crystals, $\varphi_{\text {TY }}=0.165$. Smaller yields are found for concentrated solutions of DMT in organic solvents ${ }^{19}$. In this case the dimerization can be partially quenched by triplet quenchers. This is consistent with the fact that organic solvents disrupt the association between the pyrimidines. The ratio of isomers in the quenched portion is the same as that obtained when the dimerization is sensitized by triplet energy donors. The unquenched portion has a different product ratio. These results indicate that different paths exist for dimer formation in aggregates and in dilute solution.

The situation which prevails in aggregates of thymine and uracil can be understood from the results of studies on photodimerization of oriented monomers.

Wang ${ }^{20}$ first suggested that the reason for the high efficiency $\left(\varphi_{\hat{\mathrm{TT}}} \sim 1\right)$ of photodimerization of thymine in ice where only the chh dimer is formed is that, in freezing, microcrystals of thymine hydrate are formed in which neighbouring thymines are parallel and suitably placed for dimerization. Such a crystal structure was found by Gerdil ${ }^{21}$. Eisinger and Shulman ${ }^{22}$ examined frozen water solutions of thymine at $80^{\circ} \mathrm{K}$ excited with $280 \mathrm{~nm}$ light and found that they do not emit. This is in contrast to thymine in an ethylene glycol water glass where it is dispersed and a fluorescence yield of 0.2 is observed. This correlation between the fluorescence quenching and efficient dimerization shows that a process connected to dimer formation quenches the singlet state.

Eisinger and Lamola ${ }^{23}$ were able to obtain a clearer picture in their studies of oriented monomer pairs formed by breaking dimers in a rigid matrix. 


\section{A. A. LAMOLA}

Various isomeric dimers of $\left(\sim 10^{-3} \mathrm{M}\right)$ DMT were dissolved in EGW and the sample cooled to $80^{\circ}$ where a clear glass is formed. Irradiation with 248 $\mathrm{nm}$ light was used to break some of the dimers into pairs of monomers which remain suitably positioned to reform the dimers because of the rigidity of the matrix. The absorption spectra of 'broken dimers' or monomer pairs exhibit exciton splitting and a distance between the transition dipoles in the broken cis-syn-dimer was calculated from the simplest exciton model to be $2.8 \AA$. The broken dimers do not fluoresce but redimerize with a quantum yield of $1.0 \pm 0.1$ ! It is interesting that the quantum yield for breaking the dimers is also close to unity.

The simplest interpretation of the results for thymine crystals and broken dimers is that the fluorescence quenching and efficient dimerization are directly coupled. That is, the excited singlet goes on to form dimer faster than it does anything else. One can imagine this occurring in two steps by way of an excimer intermediate. Interaction at the singlet level leading to efficient dimer formation is not expected if diffusion or conformational changes requiring more than about $10^{-10} \mathrm{sec}$ are necessary to align the second monomer.

An alternative explanation is that the interaction between the two thymines in the excited singlet state leads to totally efficient intersystem crossing and then the triplet goes on to form the dimer. Although this explanation is probably not correct it cannot be excluded because it is known that intersystem crossing is more efficent in thymine aggregates than in isolated thymines. The important point is that whether or not the triplet state is an intermediate, efficnent $(\varphi \sim 1)$ dimer formation appears to require favourable juxtaposition of the monomer units during the singlet state lifetime.

The photodimerization of pyrimidines in nucleic acids can occur by way of the triplet state since the reaction can be sensitized with triplet donors (see below). However, this tells us nothing of what the mechanism is when the light is absorbed directly by the nucleic acid. Before discussing the experimental data concerning the excited precursor question it is interesting to consider what can be said about dimerization in the dinucleotides and polynucleotides from what has been presented above. One quickly realizes that no strong conclusions can be drawn because none of the systems already mentioned are proper models for two neighbouring pyrimidines in a stacked polynucleotide or in DNA. The Watson-Crick DNA structure provides for a 36 angle between the neighbouring thymines whose parallel molecular planes are spaced $3.4 \AA$ apart. Thus neighbouring thymines in DNA or in a stacked dinucleotide like TpT are neither like thymine in dilute aqueous solution nor like the thymines in a broken dimer where they are very nicely juxtaposed for efficient dimer formation.

The quantum yield for formation of photodimers in $\mathrm{TpT}^{24}$ or in $\mathrm{DNA}^{25}$ is about 0.01 or some twenty times larger than the limiting quantum yield in dilute thymine solutions and one hundred times smaller than the yield in thymine crystals or 'broken dimers'. Photodimers are formed in concentrated aqueous solutions of thymine where aggregates are present with a yield of $0.04^{26}$. The ratio of stereoisomers formed is very similar to the ratio formed in $\mathrm{TpT}$ so that concentrated solutions of thymine may be suitable models for TpT. However, this provides no help in deciding whether the 
photodimerization is a singlet state reaction controlled by the distribution of possible juxtapositions of neighbouring thymine molecules or whether the presence of a neighbouring thymine induces intersystem crossing followed by dimerization from the triplet state.

Only cis-syn-dimers are produced in native DNA and in well stacked homopolynucleotides ${ }^{27}$. Of course, the geometry of the polymer excludes the formation of the other cis-fused stereoisomers.

Rahn has measured the rate and extent of dimer formation in poly $U$ in a frozen glass and observed that the number of dimers saturated with increasing dosage but that additional dimers can be formed if the glass is allowed to melt and is then refrozen ${ }^{28}$. This observation is consistent with a 'geometry-limited' dimerization mechanism. The polynucleotide in the frozen glass preserves its geometry in which only a certain fraction of the pyrimidine pairs are in a suitable juxtaposition for dimerization. The same view has been taken of thymine dimerization in DNA and is supported by the observation that the photodimer yield is virtually constant below the DNA melting point but drops considerably at temperatures above it $^{29}$.

A very nice experiment showing the importance of stacking for efficient photodimer formation is that of Wacker and Lodeman who measured the relative yield of photodimerization in $\mathrm{TpT}$ in various solvents ${ }^{30}$. They found a monotonic relation between the dimer yield and the free energy difference between the stacked and unstacked forms of TpT, which varied with the solvent. The dimer yield is higher in those solvents in which stacking is favoured.

Experiments with added quenchers have provided additional insight into the excited precursor problem. The addition of specific triplet quenchers such as dienes and paramagnetic metal ions does not affect the rate of photodimer production in $\mathrm{DNA}^{31,32}$ or in $\mathrm{TpT}^{33}$. This is consistent with a model in which dimerization is a singlet state process. However, it may simply reflect a dimerization via the triplet state which occurs at a rate much faster than the quenching rate.

The Sutherlands have shown that acridine orange, methyl green, ethidium bromide and chloroquine reduce the dimer yield in DNA ${ }^{32}$. They also observed DNA sensitized dye fluorescence and have presented evidence that the sensitized dye fluorescence and dimer inhibition is due to Förstertype singlet-singlet transfer from the DNA to the dye. More recently these investigators measured the dependences of the efficiencies of dimerization inhibition and dye fluorescence upon the wavelength of the exciting light. They found that the efficiency of dimer inhibition by ethidium bromide is greater at shorter wavelengths of the exciting light. On the other hand, the efficiency of the sensitized dye fluorescence is wavelength independent. The data suggest that at excitation energies higher than that necessary to excite the lowest vibrational levels of the DNA singlet state singlet-singlet transfer cannot account for all the dimer quenching. Intersystem crossing is the only documented wavelength dependent process in DNA constituents and so the Sutherlands suggest that the model proposed by Brown and Johns for poly $U$ is applicable to DNA. In this model, dimers are formed directly from the excited singlet state as well as via the triplet state with triplets 


\section{A. A. LAMOLA}

arising from intersystem crossing from higher vibrational levels of the excited singlet state.

Photodimer yields and kinetics have been used in the interpretation of structure for polynucleotides and are of particularly high interest in studies of tRNA. Some results of poly $U$ are mentioned in the section on photohydration. One other example is mentioned here. Cerutti and co-workers ${ }^{34}$ have shown that while pyrimidine dimers are formed upon irradiation of solutions of the RNA from R17 phage, no dimers are formed upon extended irradiation of intact virus particles. It was concluded that an intimate association of the RNA with the coat proteins suppresses the formation of photodimers. It is thought that the suppression is due to geometric factors; however, excited state quenching by the protein has not been ruled out.

\section{Sensitized pyrimidine dimers in polynucleotides}

The energy levels for the lowest lying excited singlet and triplet levels of the common nucleotides are shown schematically in Figure 5 together with those of acetone and acetophenone ${ }^{35}$. On the basis of the order of these

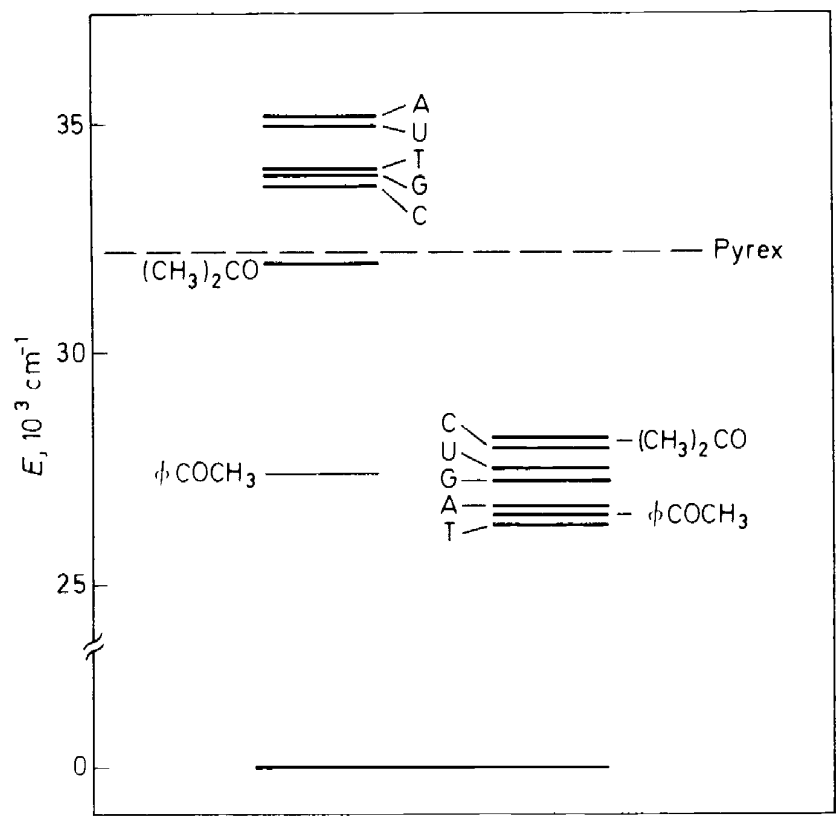

Figure 5. The energies necessary to excite the lowest excited singlet states and lowest triplet states of the common nucleotides and those of acetone and acetophenone.

levels it is possible to excite triplet states in DNA by means of triplet excitation transfer from acetone or acetophenone which can be excited with light not absorbed by the DNA, e.g. $313 \mathrm{~nm}$. The triplet of acetone can transfer to all the bases while that of acetophenone can transfer only to thymine. 
Both acetone and acetophenone have high intersystem crossing efficiencies $\left(\varphi_{\text {isc }} \sim 1.0\right)$ but unfortunately the triplets of these molecules are short lived $\left(\tau \leqslant 10^{-6}\right)$ in water solution (even in the absence of oxygen) so that the efficiency of triplet transfer to the bases in DNA is expected to be low. One can nevertheless observe photochemical reactions in DNA which are sensitized by acetophenone ${ }^{36}$ and acetone ${ }^{37}$. These occur at convenient rates and what is more important give pyrimidine dimers as the only major products. In contrast to this, the direct irradiation of DNA $(254 \mathrm{~nm})$ leads to a variety of products in comparable yields.

Since the acetophenone triplet can transfer only to thymine it is expected that only products involving thymine or the sensitizer would be found. The most complete product analysis has been performed on E. coli DNA irradiated at $\lambda \geqslant 313 \mathrm{~nm}$ in water solution $(0.1 \mathrm{M}$ phosphate buffer $)$ in the presence of acetophenone $\left(10^{-2} \mathrm{M}\right)^{38}$. The results are shown in Table 2 and

Table 2. Comparison of the initial yields of photoproducts detected in E. coli DNA as a result of direct irradiation at $254 \mathrm{~nm}$ and sensitization by acetophenone $\left(10^{-2} \mathrm{M}\right)$ and acetone $(1 \mathrm{M})$ at wavelengths greater than $313 \mathrm{~nm}$. The rate of production of TiT is taken as unity. The data for acetophenone are from reference 40 and those for acetone are from reference 37 .

\begin{tabular}{|c|c|c|c|}
\hline \multirow{2}{*}{ Material } & \multirow{2}{*}{ Direct } & \multicolumn{2}{|c|}{ Sensitized } \\
\hline & & Acetophenone & Acetone \\
\hline$\hat{\text { TT }}$ & $(1.0)$ & (1.0) & $(1.0)$ \\
\hline$\hat{C T}$ & 0.8 & 0.03 & 0.14 \\
\hline$\hat{\mathrm{CC}}$ & 0.2 & $<0.0025$ & $\sim 0.01$ \\
\hline 5,6-Dihydrothymine & 0.1 & 0.02 & \\
\hline Cytosine photohydrate & 0.3 & $<0.003$ & \\
\hline $6-4^{\prime}-\left[\right.$ Pyrimidine $-2^{\prime}$-one $]$-thymine & 0.1 & $<0.0025$ & $<0.002$ \\
\hline Sensitizer addition & & $<0.02$ & \\
\hline
\end{tabular}

show a striking reduction in photoproducts containing cytosine compared to direct irradiation. Some results for acetone sensitization ${ }^{39}$ are also shown in the table and show an increase in cytosine dimers relative to acetophenone sensitization.

Meistrich, Lamola and Gabbay ${ }^{39}$ have used two different derivatives of acetophenone to introduce thymine dimers into T4 phage. Using this technique Mejstrich ${ }^{40,41}$ studied the inactivation of the phage due to the presence of TT and Meistrich and Shulman and Drake investigated the mutagenic effect of $\hat{T T}^{42,43}$. Mennigmann and Wacker ${ }^{44}$ have used acetone to sensitize the inactivation of an E. coli mutant. Chambers et $a l^{45}$ have used acetone sensitization to simplify the photochemistry of tRNA. This is very useful since only pyrimidine dimers are formed in this way and the number of dimerizable sites in a particular tRNA is small.

The distribution of dimers in DNA might be quite different for direct irradiation compared to triplet state sensitization because of the several base-base processes which occur at the singlet but not at the triplet level. For example, one might expect less rapid relaxation and a higher photochemical yield in A-T rich regions compared to $\mathrm{G}-\mathrm{C}$ regions after direct 


\section{A. A. LAMOLA}

relaxation. Sensitization with acetone, on the other hand, should lead to a rather uniform distribution of triplet states. Brunk ${ }^{46}$ has recently supplied some data relevant to this point.

\section{Photohydration of pyrimidines}

The photohydration reactions of uracil and cytosine derivatives have been known longer than any of the other photoreactions of the common pyrimidines ${ }^{47}$. Despite this long history and considerable efforts the mechanism of photohydration remains obscure ${ }^{4}$.

In these reactions the elements of water are added across the 5,6-double bond; the $-\mathrm{OH}$ group goes at the 6-position. The reaction occurs for uracil and cytosine and many of their derivatives but not for thymine. The photohydrates are thermally unstable and lose water in an acid catalysed reaction to reform the pyrimidines. This characteristic is very useful in that in systems in which both photohydration and photodimerization occur the fraction of photohydration can be assessed by subsequent heating to revert the photohydrates but not the dimers ${ }^{48}$.

The photohydration reactions most certainly do not involve a triplet state intermediate: in dilute solutions of uracil photodimerization may be quenched by triplet state quenchers leaving the photohydration reaction unaltered $^{15,49,50}$. The photodimer yield increases with decreasing exciting light wavelength as does the intersystem crossing, but the photohydration yield is independent of the wavelength. Triplet state sensitizers induce the formation of photodimers of uracil and cytosine but no photohydrates are formed $^{45,51,52}$. Finally, the photohydration reactions exhibit a negative temperature dependence, similar to that of fluorescence ${ }^{3}$.

The photohydration reactions are $\mathrm{pH}$ dependent ${ }^{4,53}$. The ' $\mathrm{pKs}$ ' relevant to the reaction ( $\sim 5$ for 3 -alkyl uracils) are different from the known $\mathrm{pKs}$ for the ground state species. The results indicate that neutral excited species react more efficiently than ionized species.

It would seem that a simple interpretation of the data is as follows: It is the excited singlet state of the pyrimidine which reacts and the $\mathrm{pH}$ dependence reflects the $\mathrm{p} K$ of this state. One can then write down a number of mechanisms for the addition of the water, the most attractive involving protonation at the 4-carbonyl oxygen as the primary step.

The inadequacy of this explanation appears when one considers the observed lifetimes of the singlet states which are of the order of $10^{-12} \mathrm{sec}$. It does not seem possible for a protonation-deprotonation equilibrium to be achieved in such a short time at low proton concentrations, so that the $\mathrm{pH}$ dependence becomes difficult to explain. Furthermore the primary process must involve reaction with water since it is the only species present at sufficiently high concentration to react with the short-lived singlet state.

Further confusion is supplied by the observation by Burr that at $\mathrm{Eu}^{3+}$ concentrations high enough significantly to quench the $10^{-12}$ sec-lived fluorescent singlet state referred to above, the photohydration of UMP is not affected ${ }^{53}$. It appears that the singlet state of UMP which is capable of energy transfer to $\mathrm{Eu}^{3+}$ is not the state responsible for the photohydration.

The relative quantum efficiencies for photohydrate formation in many dinucleotides and polynucleotides have been measured in recent years. 
Some data, mostly due to Grossman ${ }^{54}$, for the photohydration of cytosine moieties is given in Table 3 .

The initial photohydrate yield in the single stranded poly $\mathrm{C}$ is about three times lower than the yield for the monomer. After about half of the cytosines are reacted in the polymer the quantum yield goes to a value which is

Table 3. Cytosine photohydrate yields*

\begin{tabular}{lclc}
\hline \multicolumn{1}{c}{ Material } & $\varphi \times 10^{3}$ & \multicolumn{1}{c}{ Material } & $\varphi \times 10^{3}$ \\
\hline CMP-5' $^{\prime}$ & 16.2 & Poly C: Poly I & 2.7 \\
Poly C & $6.8(13) \dagger$ & DNA & 1.6 \\
Poly C $(90 \%$ EG) & 15.6 & DNA (single stranded) & 5.1 \\
\hline
\end{tabular}

* From reference 54

$\dagger$ The yield increases to the value in parentheses after about half of the groups are reacted (see references 6 and 55 ).

about the same as the initial yield for poly $\mathrm{C}$ in 90 per cent ethylene glycol which is close to the value for the monomer. The low initial yield for poly $\mathrm{C}$ in water undoubtedly reflects secondary structure which is thought to involve significant base stacking. Lomart and $\mathrm{Fresco}^{6,55}$ blame the low yield of hydration on the limitation imposed upon accessibility of water molecules by the base stacking. While this may well be the explanation it should be kept in mind that the stacking could change the lifetimes and electron distribution in the excited singlet state. But as was mentioned in the section on photophysics the rate of these base-base interactions at room temperature relative to the monomer lifetimes is unknown.

Photohydrate production in the double stranded poly C:poly I (I is inosine) is even less efficient than in poly $\mathrm{C}$. This may reflect still greater limitation to water accessibility due to the even more rigid structure or may reflect competition from electronic relaxation processes involving the $\mathrm{C}-\mathrm{I}$ hydrogen bonding as in C-G pairs. The yield of cytosine photohydration is small even in DNA. The yield for single-stranded DNA in which significant base stacking remains is similar to that for poly $\mathrm{C}$.

The yields of photoproduct formation in various uracil containing compounds are given in Table $4^{56,57}$. The fractions which are thermally reversible represent the photohydrate fractions; the remainders are mostly cyclobutane dimers.

Table 4. Uracil photoproduct yields*†

\begin{tabular}{lrrlcc}
\hline & $\varphi \times 10^{3}$ & $\%$ tr $\ddagger$ & & $\varphi \times 10^{3}$ & $\% \operatorname{tr\ddagger }$ \\
\hline UMP & 21 & $>90$ & GpUpA & 7 & $>90$ \\
UpUp & 70 & 85 & GpApU & 5 & $>90$ \\
poly U & 55 & 65 & poly U: poly A $2.5(3.5) \S$ & $100(80)$ \\
ApUp & 7 & 100 & & & \\
\hline
\end{tabular}

\footnotetext{
* From reference 56 .

† See also reference 6 .

\$ The percentage of uracil photoproducts reversed by heating.

$\$$ The yield increases to the value in parentheses after about half of the groups are reacted.
} 


\section{A. A. LAMOLA}

Photohydrate formation increases in UpUp compared to the monomer and the yield in the deoxydinucleotide is even higher. Photohydration in poly $\mathrm{U}$ proceeds at about the same rate as in the dinucleotide. This is consistent with other measurements which indicate that there is little or no base stacking in UpUp or in poly U. Photodimer formation kinetics indicate the same conclusion. The quantum yield of dimer formation, after statistical correction, is similar for the polymer and dinucleotide. Addition of solvents which tend to denature stacked polymers does not affect the dimer yield.

The photohydrate yield decreases in ApUp, GpUpA and GpApU compared with $U p$. It may be that the stacking with the neighbouring purine limits water accessibility, but energy transfer to or exciplex formation with the purine neighbours are additional possibilities for the reduced yield.

The yield of photohydration is further reduced on going to poly $U$ : poly A. This may reflect excited state quenching due to the hydrogen bonding or simply further limitations on water accessibility.

\section{'Spore Product' and dihydrothymine}

Two products isolated from u.v.-irradiated DNA involve the saturation of the 5,6-double bond of thymine in a way suggestive of free radical intermediates. These are 5,6-dihydrothymine $\left(\mathbf{T H}_{2}\right)^{58}$ and the so-called spore<smiles>CC1CNC(=O)NC1=O</smiles>

$\mathrm{TH}_{2}$<smiles>CC1(Cc2c[nH]c(=O)[nH]c2=O)CNC(=O)NC1=O</smiles>

$\mathbf{s p}$

product (sp) which is produced upon irradiation of spores and of dried DNA ${ }^{59}$. Both of these products could arise from the free radicals, 5,6-dihydrothym-5-yl and 5-uracilmethyl, observed upon radiolysis of thymine monohydrate.<smiles>CC1=C(C)NC(=O)NC1</smiles>

5,6-dihydrothym-5-yl<smiles>Cc1c[nH]c(=O)[nH]c1=O</smiles>

5-uracilmethyl

Hydrogen abstraction by 5,6-dihydrothym-5-yl would give $\mathbf{T} \mathbf{H}_{2}$, and coupling of the two radicals would give sp. These radicals could arise from the anion radical and cation radical of thymine. Protonation of the anion radical at carbon-6 gives 5,6-dihydrothym-5-yl and loss of a methyl proton from the cation radical gives 5-uracilmethyl. If the radical anion and radical cation were produced as neighbours proton transfer from the methyl group 
of the cation to the 6-carbon of the anion might give the radical pair which could couple to give sp.

It is possible to generate the anion radical and cation radical of thymine and also both 5,6-dihydrothym-5-yl and 5-uracilmethyl with u.v. light. All four species can be observed upon photolysis of thymine in very acidic or very basic aqueous glasses at $77^{\circ} \mathrm{K}^{60}$. Cation production probably involves photoionization of thymine by a two photon process with the triplet state as an intermediate. The photoejected electrons can be captured by other thymine molecules to form the anion radicals. Proton transfer to or from solvent leads to the neutral radicals.

The dependence of the quantum yield of sp on the light intensity ought to be determined. The two photon photoionization mechanisms mentioned above lead to a quadratic dependence of the yield upon intensity. A one photon electron transfer between neighbouring bases is also a possibility.

Dried DNA and DNA in spores has a disordered structure and few photodimers are formed but $\mathbf{s p}$ production observed. $\mathbf{s p}$ is also formed efficiently in frozen solutions of native DNA at $-100^{\circ} \mathrm{C}^{61}$. $\mathbf{T H}_{2}$ is produced as a result of irradiation under these conditions ${ }^{62}$ and also in DNA in solution at room temperature where sp is not produced. It may be that both structural factors and water availability govern the efficiency of production of $\mathbf{s p}$ and $\mathbf{T H}_{2}$.

\section{4-Thiouracil-cytosine adduct}

Several tRNAs from E. coli possess a 4-thiouridine $\left(\mathrm{s}^{4} \mathrm{U}\right)$ moiety at position 8. This nucleotide exhibits an intense absorption band near $335 \mathrm{~nm}$. Irradiation near $335 \mathrm{~nm}$ of these $\mathrm{s}^{4} \mathrm{U}$ containing tRNAs which also psssess a cytosine base at position 13 leads to the formation of an adduct between the $s^{4} U$ and $\mathrm{C}^{63}, 64$. Bergstrom and Leonard ${ }^{65}$ have proposed that the adduct is 5-(4'-pyrimidin-2'-one)-4-thiouracil ( $\left.\mathrm{PO}-\mathrm{s}^{4} \mathrm{U}\right)$ which arises after rearrangement and loss of hydrogen sulphide from the primary photochemical product, the thietane.<smiles>[R]N1CCC(N)=NC(=O)N1</smiles><smiles>[R]n1ccc(=S)[nH]c1=O</smiles>

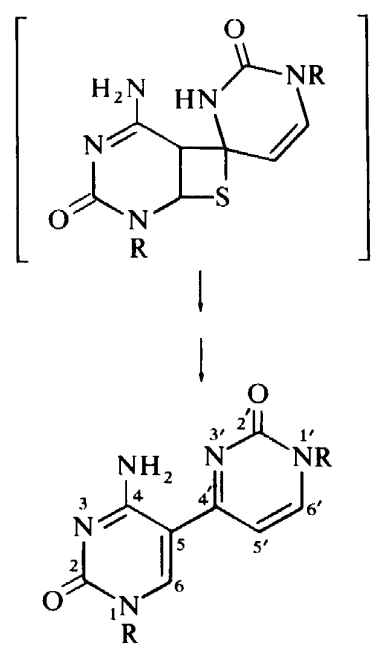




\section{A. A. LAMOLA}

What does the occlirrence of this reaction tell about the structure of the tRNAs? Obviously the formation of the product requires a tertiary structure which places the $\mathrm{C}=\mathrm{S}$ bond of the $\mathrm{s}^{4} \mathrm{U}$ close to and perhaps necessarily approximately parallel to the 5,6-double bond in the cytosine moiety. It has recently been shown that the intact active tertiary structure of these tRNAs is a requirement for the photoaddition reaction ${ }^{66}$. Unfortunately, the quantum yield is low, $\sim 0.005$. Thus there is the question: Does the tRNA structure always have the $s^{4} U$ and $C$ nicely juxtaposed for a reaction which has an intrinsically low quantum yield: or does the reaction of nicely juxtaposed groups go with high efficiency with the low yield reflecting a dynamic situation in which the groups only infrequently assume the proper relative orientation? Knowledge of the excited state precursor(s) for this reaction as well as the lifetimes of these states is important for further interpretation. At this time there is no conclusive information about these points.

\section{Pseudouridine $(\psi)$ photocleavage}

Irradiation of tRNAs containing $\psi$ with $254 \mathrm{~nm}$ light leads to chain scission at the $\psi \operatorname{sit}^{67}$. 5-Formyluracil is obtained as a fragment so that the cleavage occurs across the ribose ring. The reaction does not involve the triplet state of the base since it does not occur upon triplet sensitization with acetone. The quantum efficiency which is about $0.03(254 \mathrm{~nm})$ seems to be independent of the particular sequence of bases around the $\psi$ site $^{68}$. Whether or not the quantum yield is wavelength dependent is not known. The mechanism for this curious and useful photoreaction should be very interesting.

\section{PO-T}

An adduct derived from cytosine and thymine which has been isolated from irradiated DNA has been shown by Wang and Varghese to be 6-4'[pyrimidine-2'-one thymine] $(\mathrm{PO}-\mathrm{T})^{69,70}$.<smiles>Cc1c(-c2ccnc(O)n2)[nH]c(=O)[nH]c1=O</smiles>

It is reasonable to assume that PO-T is the hydrolysis product of a photoadduct having the following structure.<smiles>CN1C=CC2(NC1=O)NC1(C)C(=O)NC(=O)N(C)C21</smiles> 
An intriguing proposal for the product of the azetane involves phototautomerization in the G-C base pair; which may also explain the lack of fluorescence from excited G-C pairs:
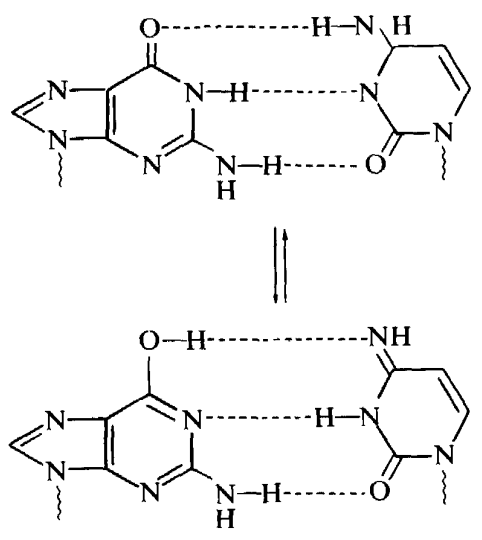

The imino double bond of the excited tautomeric form of cytosine could then add to the 5,6-double bond of a neighbouring thymine:<smiles></smiles>

This mechanism predicts that PO-T is not formed efficiently in singlestranded DNA. This has not been tested.

\section{CONCLUSIONS}

Studies of the mechanisms and structural requirements for the photoreactions in polynucleotides are complicated by the low yields of the reactions and the essential lack of luminescence from the nucleotides in aqueous solution near room temperature. The low yields of chemistry and luminescence are due mostly to the competition by very fast $\left(\sim 10^{12} \mathrm{sec}^{-1}\right)$ radiationless relaxation processes intrinsic to the monomers which degrade the excited singlet states. Thus, limited success has been met with in attempts to obtain structural details from photochemical studies. On the other hand the use of photochemical yields in an empirical way to indicate the occurrence of 


\section{A. A. LAMOLA}

conformational changes has been successful. The most successful use of photochemical reactions in polynucleotides has been for making specific structural modifications for structure-function studies.

\section{REFERENCES}

1 A. D. McLaren and D. Shugar, Photochemistry of Proteins and Nucleic Acids, p 162. MacMillan: New York (1964).

${ }^{2}$ K. C. Smith, in Photophysiology, ed. by A. C. Giese, Vol. II, p 329, Academic Press: New York (1964).

3 J. K. Setlow, in Comprehensive Biochemistry, ed. by M. Florkin and E. H. Stotz, Vol. 27, p 157. Elsevier: Amsterdam (1967).

4 J. G. Burr, Advanc. Photochem. 6, 193 (1968).

5 R. B. Setlow, Prog. Nucleic Acid Res. Mol. Biol. 8, 137 (1968).

6 A. J. Lomant and J. R. Fresco, Prog. Nucleic Acid Res. 12, 1 (1972).

${ }^{7}$ P. C. Hanawalt, in An Introduction to Photobiology, ed. by C. P. Swanson. Prentice Hall: Englewood Cliffs, New Jersey (1969).

8 This section is a condensation of the review, J. Eisinger and A. A. Lamola in Excited States of Proteins and Nucleic Acids, ed. by R. F. Steiner and 1. Weinryb, p 107. Plenum Press: New York (1971). Primary references can be found therein.

${ }^{9}$ M. Daniels and W. Hauswirth, Science, 171, 675 (1971); W. Hauswirth and M. Daniels, Photochem. Photobiol. 13. 157 (1971).

10 Calculations connected with the model first presented in reference 8 contained errors. These have been corrected and an expanded model is given in reference 11 .

11 M. Gueron, J. Eisinger and A. A. Lamola in Principles of Nucleic Acid Chemistry, ed. by P. O. P. T'so. Academic Press; New York (to be published, 1972).

12 J. Eisinger, B. Feuer and T. Yamane. Proc, Nat. Acad. Sci., Wash. 65, 638 (1970).

13 J. Eisinger, B. Feuer and T. Yamane, Nature, London, 231, 126 (1971);

J. Eisinger, Biochem. Biophys. Res, Commun. 43, 854 (1971).

14 K. Beardsley and C. R. Cantor, Proc. Nat. Acad. Sci., Wash. 65, 39 (1970).

15 A. A. Lamola and J. P. Mittal, Science, 154, 1560 (1966).

16 C. L. Greenstock, I. H. Brown, J. W. Hunt and H. E. Johns, Biochem. Biophys. Res. Commun. 27, 431 (1967).

17 P. Wagner and D. J. Bucheck, J. Amer. Chem. Soc. 92, 181 (1970).

${ }_{18}$ R. Lisewski and K. L. Wierzchowski, Molec. Photochem. 3, 231 (1971).

19 H. Morrison, A. Feeley and R. Kloepfer, Chem. Commun. 358 (1964).

20 S. Y. Wang, Nature, London, 190, 690 (1960);

S. Y. Wang, Photochem. Photobiol. 3, 395 (1964).

21 R. Gerdil, Acta Crystallogr. 14, 333 (1961).

22 J. Eisinger and R. G. Shulman, Proc. Nat. Acad. Sci., Wash. 58. 895 (1967).

23 A. A. Lamola, Molec. Photochem. 1, 209 (1969).

${ }^{24}$ H. E. Johns, M. L. Pearson, J. C. LeBlanc and C. W. Hellinor, J. Molec. Biol. 9, 503 (1964).

${ }_{25}$ H. E. Johns, S. A. Rappaport and M. Delbruck, J. Molec. Biol. 4, 104 (1962).

${ }^{26}$ G. J. Fischer and H. E. Johns, Photochem. Photobiol. 13 (1971).

27 D. Weinblum, Biochem. Biophys. Res. Commun. 27, 384 (1967).

${ }^{28}$ R. O. Rahn, Science, 154, 503 (1966).

29 J. L. Hosszu and R. O. Rahn, Biochem. Biophys. Res. Commun. 29, 327 (1967).

30 A. Wacker and E. Lodeman. Angew. Chem. Internat. Ed. 4, 150 (1965).

31 J. Eisinger, R. G. Shulman and T. Yamane, Proceedings of the Gatlinberg Conference (W. Snipes, ed.), Nuclear Science Set 43, National Academy Sciences and National Research Council (1966).

32 B. M. Sutherland and J. C. Sutherland, Biophys. J. 8, 490 (1969); 9, $1(145$ (1969).

33 J. Eisinger and A. A. Lamola, Biochem. Biophys. Res. Commun. 28, 558 (1967).

34 P. Cerutti, N. Miller, M. Pleiss, J. Remsen and W. Ramsay, Proc. Nat. Acad. Sci., Wash. 64, 731 (1969);

J. F. Remsen, N. Miller and P. Cerutti, Proc. Nat. Acad. Sci., Wash. 65, 470 (1970)

${ }_{35}$ A. A. Lamola, M. Gueron, T. Yamane, J. Eisinger and R. G. Shulman, J. Chem. Phys. 47, 2210 (1967). 


\section{PHOTOCHEMISTRY AND STRUCTURE IN NUCLEIC ACIDS}

36 A. A. Lamola and T. Yamane, Proc. Nat. Acad. Sci., Wash. 58, 443 (1967).

37 R. Ben-Ishai, E. Ben-Hur and Y. Hornfield, Israel J. Chem. 6, 769 (1968).

38 A. A. Lamola, Photochem. Photobiol. 9, 291 (1969).

39 M. Meistrich, A. A. Lamola and E. Gabbay, Photochem. Photobiol. 11, 169 (1970).

40 M. Meistrich and A. A. Lamola, J. Molec. Biol. 66, 83 (1972).

41 M. Meistrich, J. Molec. Biol. 66, 97 (1972).

42 M. Meistrich and R. G. Shulman, J. Molec. Biol. 46, 157 (1969).

43 M. Meistrich and J. W. Drake, J. Molec. Biol. 66, 107 (1972).

44 H.-D. Mennigmann and A. Wacker, Photochem. Photobiol. 11, 291 (1970).

45 R. W. Chambers, H. P. Waits and K. A. Freude, J. Amer. Chem. Soc. 91, 7203 (1969).

46 P. Brunk, 16th Annual Biophysical Society Meeting, Abstract p 236a, Toronto, Canada (1972).

47 R. L. Sinsheimer and R. Hastings, Science, 110, 525 (1949).

48 Cytosine products with saturated 5,6-double bonds are unstable towards deamination. Thus cytosine photohydrate may be hydrolysed to uracil photohydrate and cytosine photodimers may be hydrolysed to uracil photodimers.

49 C. L. Greenstock, I. H. Brown, J. W. Hunt and H. E. Johns, Biochem. Biophys. Res. Commun. 27, 431 (1967).

50 J. C. Burr, B. R. Gordon and E. H. Park, Photochem. Photobiol. 8, 73 (1968).

51 C. L. Greenstock and H. E. Johns, Biochem. Biophys. Res. Commun. 30, 21 (1968).

52 A. A. Lamola, unpublished results.

53 J. G. Burr, to be published.

54 L. Grossman, Photochem. Photobiol. 7, 727 (1968).

55 A. J. Lomart and J. R. Fresco, J. Molec. Biol. 66, 49 (1972).

56 D. Shugar in The Nucleic Acids, ed. by E. Chargaff and J. N. Davidson. Vol. III, p 39. Academic Press: New York (1960)

57 For additional data and interpretation see the review by Lomant and Fresco, reference 6.

58 T. Yamane, B. J. Wyluda and R. G. Shulman, Proc. Nat. Acad. Sci., Wash. 58, 439 (1967).

59 J. E. Donnellan Jr and R. B. Setlow, Science, 149, 308 (1965);

A. J. Varghese, Biochem. Biophys. Res. Commun. 38, 484 (1970).

60 M. D. Sevilla, J. Phys. Chem. 75, 626 (1971); and references therein.

61 R. O. Rahn and J. L. Hosszu, Photochem. Photobiol. 7, 637 (1968).

62 The 5,6-dihydrothym-5-yl radical is observed on irradiation of frozen solutions of DNA: P. S. Pershan, R. G. Shulman, B. J. Wyluda and J. Eisinger, Science, 148, 378 (1965).

63 A. Favre, M. Yaniv and A. M. Michelson, Biochem. Biophys. Res. Commun. 37, 266 (1969).

64 M. Yaniv, A. Favre and B. G. Barrell, Nature, London, 223, 1331 (1969).

65 D. E. Bergstrom and N. J. Leonard, Biochemistry, 11, 1 (1972).

66 A. Favre, A. M. Michelson and M. Yaniv, J. Molec. Biol. 58, 367 (1971).

67 M. Tomasz and R. W. Chambers, Biochemistry, 5, 773 (1966).

68 R. W. Chambers and coworkers, unpublished results.

69 A. J. Varghese and S. Y. Wang, Nature, London, 213, 809 (1967).

70 S. Y. Wang and A. J. Varghese, Biochem. Biophys. Res. Commun. 29, 543 (1967). 